Tanja Schwander · Hervé Rosset • Michel Chapuisat

\title{
Division of labour and worker size polymorphism in ant colonies: the impact of social and genetic factors
}

Received: 7 April 2005 / Revised: 6 June 2005 / Accepted: 6 June 2005 / Published online: 29 July 2005

(C) Springer-Verlag 2005

\begin{abstract}
Division of labour among workers is central to the organisation and ecological success of insect societies. If there is a genetic component to worker size, morphology or task preference, an increase in colony genetic diversity arising from the presence of multiple breeders per colony might improve division of labour. We studied the genetic basis of worker size and task preference in Formica selysi, an ant species that shows natural variation in the number of mates per queen and the number of queens per colony. Worker size had a heritable component in colonies headed by a doubly mated queen $\left(h^{2}=0.26\right)$ and differed significantly among matrilines in multiple-queen colonies. However, higher levels of genetic diversity did not result in more polymorphic workers across single- or multiplequeen colonies. In addition, workers from multiple-queen colonies were consistently smaller and less polymorphic than workers from single-queen colonies. The relationship between task, body size and genetic lineage appeared to be complex. Foragers were significantly larger than broodtenders, which may provide energetic or ergonomic advantages to the colony. Task specialisation was also often associated with genetic lineage. However, genetic lineage and body size were often correlated with task independently of each other, suggesting that the allocation of workers to tasks is modulated by multiple factors. Overall, these results indicate that an increase in colony genetic diversity does not increase worker size polymorphism but might improve colony homeostasis.
\end{abstract}

Keywords Size polymorphism · Heritability ·

Polyethism · Social insects $\cdot$ Formica selysi

Communicated by J. Heinze

T. Schwander $(\triangle) \cdot$ H. Rosset $\cdot$ M. Chapuisat

Department of Ecology and Evolution, Biology Building,

University of Lausanne,

1015 Lausanne, Switzerland

e-mail: Tanja.Schwander@unil.ch

Tel.: +0041-21-692-41-81

Fax: +0041-21-692-41-65

\section{Introduction}

Division of labour among workers is an important component of the organization of insect societies that largely contributes to their ecological success (Wilson 1971; Oster and Wilson 1978). Workers tend to specialise on tasks such as brood tending, foraging or colony defence (Hölldobler and Wilson 1990). To optimize colony efficiency, an adequate number of workers has to be allocated to each task, which has raised considerable interest in the factors and mechanisms influencing the behavioural specialization of workers (Oster and Wilson 1978; Beshers and Fewell 2001).

Task preference is often associated with morphological adaptations, particularly in ants (Oster and Wilson 1978; Hölldobler and Wilson 1990). The association between phenotype and task is most pronounced in species with morphologically distinct worker castes (Wilson 1976; Wetterer 1999). However, some amount of phenotype-task matching is also commonly found in species where workers do not belong to distinct castes but differ in size (Wilson 1968; Oster and Wilson 1978; Hölldobler and Wilson 1990; Waser 1998), and this association between task and morphology has been shown to result in an increased efficiency of workers (Wilson 1980; Franks 1985; Porter and Tschinkel 1985b).

Two studies have documented a significant genetic component to worker size within colonies of ants (Fraser et al. 2000; Hughes et al. 2003). These findings are in accordance with the hypothesis that genetically more diverse colonies have more variable workers, and hence a more efficient division of labour (Crozier and Page 1985; Robinson 1992). However, the heritability of worker size was low in another study (Bargum et al. 2004), and the impact of a heritable component to worker size on colony efficiency will depend on the precise relationship between task specialization, genotype and size, as well as on the regulation of worker size distribution at the colony level.

Task preference may also have a direct genetic basis, independently of size. In many species of bees, wasps and ants, workers from different maternal or paternal 
lineages tend to carry out different tasks (Stuart and Page 1991; Page et al. 1995). This genetic polyethism might come from genetic variation in the response thresholds to task-related stimuli, which can generate individual specialisation while retaining a flexible allocation of workers to tasks that permits to match the needs of the colony (Robinson 1992; Beshers and Fewell 2001; Page and Erber 2002). An increase in colony genetic diversity may therefore allow a more complete or more sensitive expression of the genetically-based division of labour, leading to a more efficient worker force (Crozier and Page 1985; Robinson 1992).

In this study, we evaluate if higher colony genetic diversity increases worker size polymorphism and thus may improve division of labor. We first test whether worker size and task preference have a genetic component within colonies of Formica selysi, an ant species that shows natural variation in the number of mates per queen and the number of queens per colony. We then examine how genetic diversity and social structure affect worker size polymorphism at the colony level, and disentangle the relative effect of size and genotype on task specialisation.

\section{Materials and methods}

\section{Study population and sampling}

The study population of $F$. selysi is located along the river Rhône between Sierre and Susten in Switzerland. The social structure of 112 colonies had previously been determined by genotyping eight to 24 workers at nine microsatellite loci (Chapuisat et al. 2004). The majority of the colonies $(57 \%)$ were headed by one singly mated queen, a few $(6 \%)$ had one doubly mated queen and the remaining (37\%) had multiple queens. Single-queen colonies and multiple-queen colonies have similar mating systems and are not genetically differentiated (Chapuisat et al. 2004).

We selected 17 colonies headed by a singly mated queen (monogyne, M1), six colonies headed by a doubly mated queen (monogyne, M2) and 20 colonies with multiple queens (polygyne, $\mathrm{P}$ ). All but one polygyne colonies had many queens per colony, with genetically-effective queen number ranging from 4.6 to 36.1 . The remaining colony, which had three matrilines and a genetically-effective queen number of 1.4 , will be referred to as oligogyne (O, colony 96). All selected colonies were more than 4 years old and had already produced winged queens or males. We estimated colony size (the number of workers per colony) with a capture-recapture method described in Sundström (1995).

To compare worker polymorphism across colonies, we collected a random sample of at least 50 workers from each of the 43 colonies. These individuals were sampled early in the morning when most workers are just below the nest surface to warm up.

To estimate the correlations between genetic lineage, task and size, we collected foragers and brood tenders in a sub- sample of 32 colonies. These colonies (16 M1, 4 M2, $1 \mathrm{O}$, $11 \mathrm{P})$ were the ones that contained a lot of small brood at the time of collection. Foragers were collected in three pitfall traps placed at $40 \mathrm{~cm}$ from the nest entrances. Brood tenders were identified by placing many workers, brood and sandy nest material into a plastic box with a black paper roof in one corner. After $15 \mathrm{~min}$, we collected brood tenders, which were the workers caring for the brood under the paper roof. All sampling took place between June and August 2002.

\section{Morphometrics}

Workers were measured to the nearest $0.001 \mathrm{~mm}$ using a stereomicroscope Nikon V-12 at a magnification of $50 \times$. In a preliminary investigation, we took six body measures from a random sample of 120 workers from each of four colonies (two M1 and two P): head width (maximum width across the eyes), head height, thorax length, scape length, tibia and femur lengths of the hind-leg. All six measures covaried isometrically, with correlation coefficients ranging between 0.72 and 0.90 . We therefore decided to use head width as a single estimate of size, because it is easy to measure and is commonly used as a dependent variable in studies of allometry (Wheeler 1991).

The repeatability of the measure of head width was high. On average, repeated measures from 50 ants differed by only $0.04 \mathrm{~mm}$ (3\% of the first measure) and were highly correlated (Pearson's moment correlation: $0.92 ; t_{48}=15.84$, $p<0.0001$ ). We measured the head width of 50-100 randomly sampled workers from each of the 43 colonies, as well as 50 foragers plus 50 brood tenders from each of the 32 colonies from the task subsample.

\section{Microsatellite genotyping}

To evaluate if there is a genetic influence on worker size and task, we genotyped 40-50 foragers and 40-50 brood tenders from each of the four monogyne colonies with a doubly mated queen, the oligogyne colony and two polygyne colonies with moderate effective queen number (3.4 and 7.5, respectively) permitting a reliable assignment of individuals to matrilines. To increase sample size in the analysis of the genetic component to worker size, we further genotyped 80-100 randomly sampled workers from two additional monogyne colonies with a doubly mated queen and one polygyne colony (effective queen number $=5$ ).

Individuals were genotyped as described in Chapuisat et al. (2004). We used nine microsatellite loci for the oligogyne and polygyne colonies: FL12, FL20 and FL21 from Chapuisat (1996), as well as FE7, FE8, FE16, FE17, FE19 and FE38 from Gyllenstrand et al. (2002). For the colonies with a doubly mated queen, we selected one or two loci permitting unambiguous discrimination of the two patrilines. 
Statistical analysis

We estimated the heritability of worker size (head width) in M2 colonies with the hierarchical design for sib analysis described in Becker (1992), which can be applied to haplo-diploid organisms by slightly modifying the quantitative genetic estimators (Liu and Smith 2000). The variance components for the estimators were calculated using a nested ANOVA model for unbalanced design, with patrilines nested in colonies (Sokal and Rohlf 1995). Heritability was quantified on the basis of the between-males withinfemales component. This measure incorporates dominance effects, but is not inflated by maternal and environmental effects.

We also tested more generally whether workers from different genetic lineages differed in size. We used a nested ANOVA model for unbalanced design, with genetic lineages nested in colonies (Sokal and Rohlf 1995). For polygyne colonies, we assigned individuals to matrilines with the maximum-likelihood methods implemented in the computer program COLONY 1.1 (Wang 2004, available at http://www.zoo.cam.ac.uk/ioz/software.htm), using colony-level allele frequencies and assuming single mating. Multiple runs of the program produced very consistent results, with the vast majority of individuals being repeatedly assigned to the same matriline. The few individuals assigned to different families in different runs were removed from the dataset. Furthermore, only matrilines with more than four members were considered to avoid the problems of low sample sizes. Because the data were not normally distributed, the significance of the differences between lineages was evaluated by randomizing individuals among patrilines or matrilines within colonies (Manly 1997).

As an estimator for worker size polymorphism within colonies, we used the unbiased variation index for head width, which is the ratio between the standard deviation and the mean, corrected for sample size (Sokal and Braumann 1980). We examined if colony genetic diversity increases size polymorphism by testing whether variation indexes for head width were greater in M2 than M1 colonies, and were negatively correlated with the nestmate relatedness in polygyne colonies estimated previously (Chapuisat et al. 2004).

To disentangle the respective relative influence of body size and genetic lineage on the task preference of workers, we tested if these factors were fully correlated or had some independent effect. We fitted logistic regressions with task (foraging or brood tending) as the response variable. We first considered the effect of one factor only (genetic lineage or size), and then added the second factor. We used a $\chi^{2}$ test to examine if adding the second factor permitted to explain significantly more deviance, which indicates that the second factor affects task specialisation independently of the first one. All statistical tests were carried out with the computer program S-Plus 2000 (Math soft Inc. 1999).

\section{Results}

Genetic component to worker size

In $F$. selysi, worker size had a unimodal distribution, and workers did not segregate into discrete morphological castes. Nevertheless, size variation was large, with head width ranging from 0.96 to $1.61 \mathrm{~mm}$.

Across the six colonies headed by one doubly mated queen, the heritability of worker head width was $0.26 \pm 0.07$ (lower limit of the $95 \%$ confidence interval $=0.15$ ) and workers belonging to different patrilines differed significantly in head width (nested ANOVA, colony effect: $p=0.0003$; effect of patriline within colony: $p=0.0006$, Fig. 1). When analysing each colony separately, worker size differed significantly between patrilines in two out of six colonies (Wilcoxon rank sum tests; $p=0.003$ and $p<0.0001$ for colony 135 and 109 , respectively; $p=0.096$, $0.805,0.189$ and 0.831 for colony $94,63,12$ and 106, respectively). Across the four polygyne and oligogyne colonies, workers from different matrilines also differed significantly in size (nested ANOVA, colony effect: $p=0.004$; effect of matriline within colony: $p=0.001$ ).

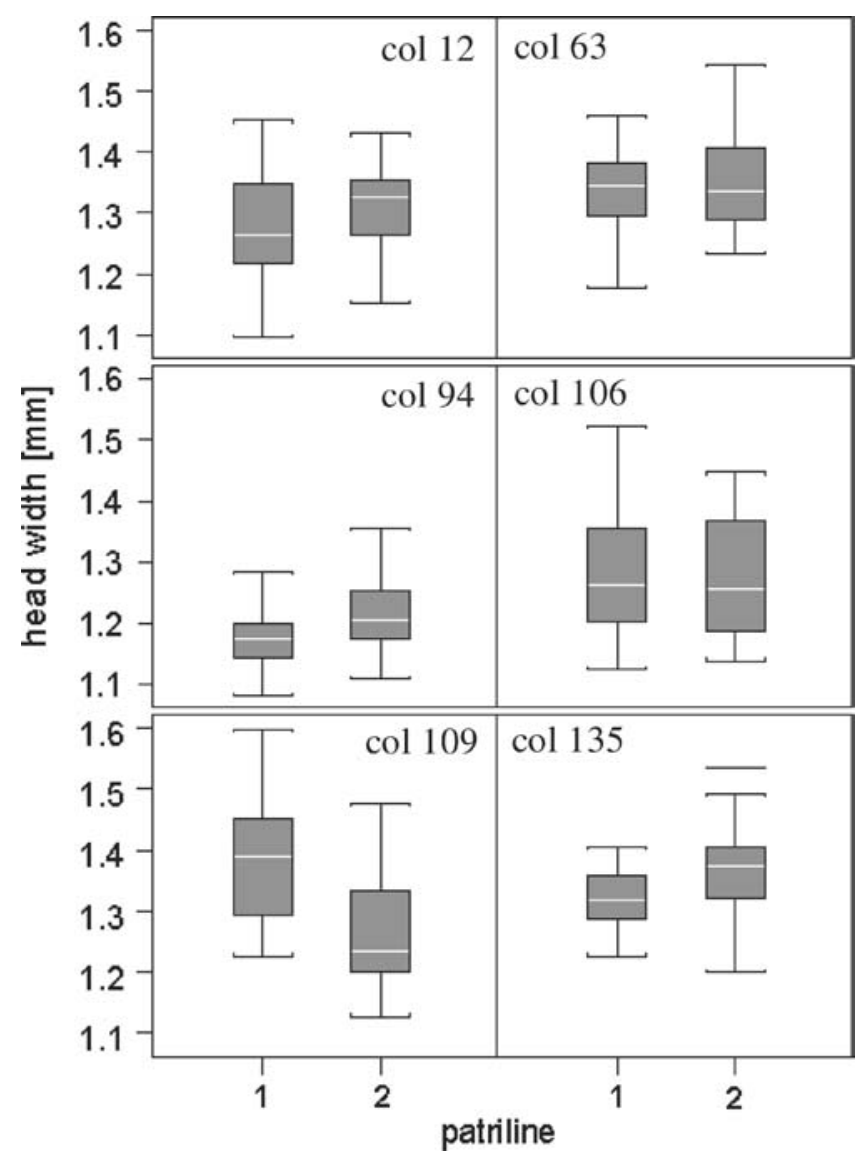

Fig. 1 Size distribution of workers from each patriline within six monogyne colonies headed by a doubly mated queen. The boxes delimit the upper and lower quartiles, and the white horizontal lines indicate the median. Whiskers include $95 \%$ of all observations 
When analysing each colony separately, worker size differed significantly between matrilines in three out of four colonies (Kruskal-Wallis tests; $p=0.0004,0.015$ and 0.036 for colony 96, 114 and 118, respectively; $p=0.242$ for colony 147).

Impact of genetic diversity, social structure and colony size on worker polymorphism

Within monogyne or polygyne colonies, increased genetic diversity was not associated with higher morphological polymorphism. In colonies headed by a singly mated queen, worker size polymorphism was not significantly lower than in colonies headed by a doubly mated queen, and the trend was in the opposite direction (one-tailed Wilcoxon rank-sum test on variation index: $W=71, p=0.923$, Fig. 2). Similarly, across polygyne colonies, colonies with lower nestmate relatedness (hence, higher genetic diversity) did not have more variable workers, the trend also being in the opposite direction (one-tailed Spearman's rank correlation: $z=1.27$, rho $p=0.31, p=0.898)$. Colony size had no significant effect on worker size (monogyne colonies: $R^{2}=0.01, F_{1,19}=0.21, p=0.65$; polygyne colonies $\left.R^{2}=0.05, F_{1,16}=0.75, p=0.40\right)$ and worker polymorphism (monogyne colonies: $R^{2}=0.12, F_{1,19}=2.49$, $p=0.13$; polygyne colonies: $R^{2}=0.09, \quad F_{1,16}=1.52$, $p=0.24$ ).

The comparison of monogyne and polygyne colonies permits to evaluate the joint impact of genetic diversity and social structure on size variation. The higher genetic diversity in polygyne colonies did not translate into increased morphological variability. To the contrary, workers from polygyne colonies were less polymorphic than workers from monogyne ones (Wilcoxon rank-sum test: $W=682, n=23$, $m=20, p<0.0001$, Fig. 2). Moreover, workers from monogyne colonies were on average about $10 \%$ larger than work- ers from polygyne ones (Wilcoxon rank-sum test: $W=709$, $n=23, m=20, p<0.0001$, Fig. 2).

Relationship between size, genotype and task

Worker size was correlated with task in both monogyne and polygyne colonies. Foragers were significantly larger than brood tenders (monogyne colonies: paired $t$-test, $t_{19}=6.84$, $p<0.0001$; polygyne colonies: paired Wilcoxon test: $V=78$, $n=12, p<0.001)$, with the mean size difference amounting to $8 \%$ and $4 \%$ in monogyne and polygyne colonies, respectively.

Genetic lineage also tended to be correlated with task. The frequency of the two patrilines differed significantly between foragers and brood tenders in three of the four colonies headed by a doubly mated queen, as well as globally (Fisher's exact test: colony 109, $p<0.0001$; colony $106, p>0.999$; colony $12, p=0.027$; colony $63, p=0.01$; Fisher's combined probability over the four colonies, $\left.\chi^{2}=34.86, d f=8, p<0.0001\right)$. In polygyne and oligygyne colonies, the frequency of matrilines differed significantly between foragers and brood tenders in one of the three colonies (Fisher's exact test: colony 96, $p=0.038$; colony $147, p=0.883$; colony $118, p=0.091$; Fisher's combined probability over the three colonies, $\chi^{2}=11.55, d f=6$, $p=0.073$ ).

Within colonies, worker size and genotype (patriline respectively matriline) often had independent effects on task, with size tending to have the strongest influence (Table 1). Size and genotype affected task independently of each other in colonies 12 and 118. Task was correlated with worker size but not with genotype in colonies 106 and 96, as well as marginally so in colony 147 . Inversely, task was correlated with genotype but not with size in colony 63. Finally, the effect of genotype on task was completely explained by the correlation with size in colony 109 (Table 1).

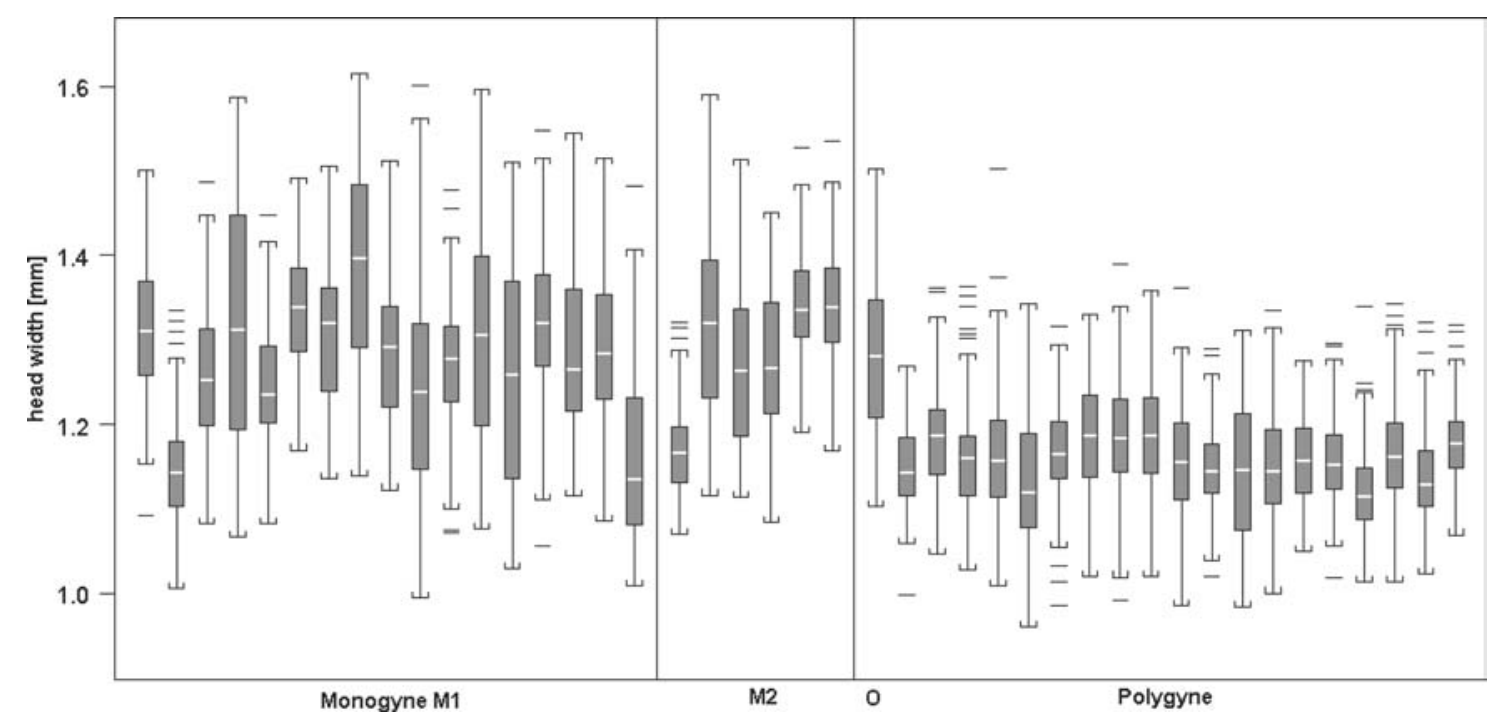

Fig. 2 Worker size distribution of all sampled colonies. Each box corresponds to one colony, which are monogyne with a singly mated queen (M1), monogyne with a doubly mated queen (M2), oligogyne (O) or polygyne, respectively 
Table 1 Logistic regression showing the relative influence of genetic lineage (patriline for the monogyne colonies 109-12, matriline for the polygyne colonies 118-96) and size (head width) on the task preference of workers $^{\mathrm{a}}$

\begin{tabular}{|c|c|c|c|c|c|}
\hline \multirow[b]{3}{*}{ Colony } & \multirow[b]{3}{*}{ Total deviance } & \multicolumn{4}{|c|}{ Deviance explained by } \\
\hline & & \multicolumn{2}{|l|}{ Model 1} & \multicolumn{2}{|l|}{ Model 2} \\
\hline & & 1. Genetic lineage & 2. Size & 1. Size & 2. Genetic lineage \\
\hline$\overline{109}$ & 121.27 & $18.50^{* * *}$ & $90.45^{* * *}$ & $72.75^{* * *}$ & $10.80 \mathrm{NS}$ \\
\hline 106 & 130.31 & $10.05 \mathrm{NS}$ & $73.34^{* * *}$ & $73.37^{* * *}$ & $10.09 \mathrm{NS}$ \\
\hline 163 & 110.90 & $18.12^{*}$ & $0.99 \mathrm{NS}$ & $10.77 \mathrm{NS}$ & $17.89^{* *}$ \\
\hline 112 & 109.50 & $15.40^{*}$ & $21.14^{* * *}$ & $28.89^{* * *}$ & $13.15^{* * *}$ \\
\hline 118 & 94.48 & $16.13^{*}$ & $12.34^{* *}$ & $9.2^{* *}$ & $19.26^{*}$ \\
\hline 147 & 43.86 & $0.90 \mathrm{NS}$ & $3.14 \mathrm{NS}$ & $3.65^{*}$ & $0.39 \mathrm{NS}$ \\
\hline 196 & 108.08 & $0.16 \mathrm{NS}$ & $28.56^{* * *}$ & $28.48^{* * *}$ & $0.24 \mathrm{NS}$ \\
\hline
\end{tabular}

${ }^{*} p<0.05,{ }^{* *} p<0.01,{ }^{* * *} p<0.0001$

${ }^{a}$ In the first model, genetic lineage was fitted first and size second. In the second model, size was fitted first and genetic lineage second

\section{Discussion}

Colony genetic diversity might improve division of labour by increasing the morphological or behavioural variation among workers (Crozier and Page 1985; Robinson 1992). Two recent studies have found a genetic component to worker size and caste in ants, suggesting that an increase in the number of breeders per colony might broaden the colony phenotype or improve caste ratios (Fraser et al. 2000; Hughes et al. 2003). However, the impact of such a genetic component on colony efficiency will critically depend on regulation processes at the level of the colony, which calls for empirical studies on how colony genetic diversity affects the colony phenotype.

We found a significant genetic component to the size of workers within colonies of $F$. selysi. In colonies headed by a doubly mated queen, the head width of workers had a heritability of $0.26 \pm 0.07$. Such size differences between patrilines most likely reflect a genetic influence on worker size, because the two lineages are produced by the same mother and share the same environment, but differ in paternal genes. Similarly, across polygyne colonies, workers from different matrilines differed in size, which also suggests a genetic influence on worker size, possibly combined with maternal and environmental effects. A genetic component to worker size was also found in the ants Camponotus consobrinus (Fraser et al. 2000) and Acromyrmex echinatior (Hughes et al. 2003), but not in Formica truncorum (Bargum et al. 2004), possibly because heritability may vary between years and social contexts (Rüppell et al. 2001; Bargum et al. 2004).

The finding of a fairly high heritability of worker size raises the question of the maintenance of genetic variability for this trait. Genetic variation at a polygenic trait such as body size may be maintained by balancing selection under pleiotropy, gene by environment interactions or fluctuation in selection pressures due to environmental variation over time or space (Bürger and Gimelfarb 2002; Turelli and Barton 2004). Alternatively, body size of individual workers may be under weak selection if the size distribution at the colony level is primarily regulated by environmental or social factors.
Indeed, size variability of the worker force at the colony level was not much affected by the genetic component to worker size within colonies. In particular, higher levels of intracolony genetic diversity did not result in increased worker polymorphism across colonies. The size polymorphism of workers was not significantly greater in colonies headed by a doubly mated queen than in colonies headed by one singly mated queen, which is in line with the results of a similar analysis in F. truncorum (Bargum et al. 2004). Across polygyne colonies of F. selysi, worker polymorphism was not significantly correlated with genetic diversity either. Moreover, in both monogyne and polygyne colonies, there was a trend for lower polymorphism in genetically more diverse colonies. Hence, our results provide no support to the hypothesis that multiple-mating or high queen numbers may be advantageous because they increase worker size polymorphism. It should, however, be noted that selection pressures may vary across species and might differ between weakly polymorphic species, such as the ones found in the genus Formica, and species with discrete worker castes.

Interestingly, $F$. selysi workers from multiple-queen colonies were consistently smaller and less polymorphic than workers from single-queen ones. This result further indicates that colony genetic diversity does not increase worker polymorphism, which is much more affected by environmental factors such as colony queen number. The pattern of variation in this population of $F$. selysi strongly suggests a direct effect of social structure on worker size and size variation, which might be a general process in ants (Bourke and Franks 1995). Indeed, workers from polygyne populations of the fire ant Solenopsis invicta are also smaller and less polymorphic than workers from monogyne populations (Greenberg et al. 1985; Goodisman and Ross 1996), and monogyne species of the genus Formica commonly have larger workers than polygyne ones (Pisarski 1981). The causes of this "polygyny syndrom" remain enigmatic. Proximately, an increase in the concentration of queen pheromones or an excess of eggs relative to the amount of available resources might explain the smaller size of workers in polygyne colonies (Porter and Tschinkel 1985a; Goodisman and Ross 1996). The higher genetic diversity in polygyne colonies might 
also buffer against environmental variation and result in a less varied colony phenotype.

The relationship between task, body size and genetic lineage appeared to be complex. Foragers were significantly larger than brood-tenders. This correlation between size and task has been documented in other weakly polymorphic species such as Leptothorax longispinosus and is likely to confer energetic or ergonomic advantages to the colony (Oster and Wilson 1978; Hölldobler and Wilson 1990; Bourke and Franks 1995).

Genetic lineage was also correlated with task specialisation: the proportion of the two patrilines differed between foragers and brood tenders in colonies headed by a doubly mated queen, and matrilines tended to be unequally represented in task groups in polygyne colonies. Such genetically based polyethism has been documented in many species of social insects (Stuart and Page 1991; Snyder 1992; Page et al. 1995; Costa and Ross 2003; Goodisman and Crozier 2003; Julian and Fewell 2004), and is likely to result from genetic variation in response thresholds (Beshers and Fewell 2001; Julian and Fewell 2004). It is not yet clear whether genetic polyethism generally results in a better and more efficient division of labour in colonies that contain more lineages, as compared to colonies with fewer lineages. However, honeybee colonies with more patrilines had less variable performances (Page et al. 1995) and more stable thermoregulation (Jones et al. 2004), suggesting that genetic diversity might improve colony homeostasis.

Our data permit us to somewhat disentangle the respective influence of size and genetic lineage on task specialisation. In $F$. selysi, genetic lineage and body size often correlated with task independently of each other. Their relative influence on task allocation varied between colonies, with size tending to have the stronger influence. In $F$. argentea, genetic subgroups correlated with task but not with size (Snyder 1992). However, the relationship between size and task was not investigated, and the power of the analysis was limited. These findings thus suggest that the allocation of workers to tasks is modulated by multiple factors having small and variable effects. A combination of controlling factors might result in a more robust division of labour, particularly during environmental perturbations. Variation in the relative importance of each factor is also expected if there is random environmental or genetic variation between colonies.

In conclusion, worker size variation in $F$. selysi colonies results from a complex interaction between genetic, social and environmental factors. We found that despite a significant heritable component to worker size, higher colony genetic diversity was not associated with increased worker size polymorphism, which provides no support to the hypothesis that high genetic diversity results in a broader colony phenotype. To the contrary, environmental or social factors that varied between monogyne and polygyne colonies, possibly coupled with a positive effect of genetic diversity on colony homeostasis, resulted in workers being less variable in colonies with higher genetic diversity.
Acknowledgements We thank C. Peter for help in the lab, S. HelmsCahan for discussions and P. Christe, R. H. Crozier, S. Helms Cahan, L. Keller and two anonymous referees for comments on earlier versions of the manuscript. This study was supported by the Swiss National Science Foundation (grant 31-61934.00 to MC)

\section{References}

Bargum K, Boomsma JJ, Sundström L (2004) A genetic component to size in queens of the ant, Formica truncorum. Behav Ecol Sociobiol 57:9-16

Becker WA (1992) Manual of quantitative genetics., 5th edn. Academic Enterprises, Pullman, WA

Beshers SN, Fewell JH (2001) Models of division of labor in social insects. Annu Rev Entomol 46:413-440

Bourke AFG, Franks NR (1995) Social evolution in ants. Princeton University Press, Princeton, NJ

Bürger R, Gimelfarb A (2002) Fluctuating environments and the role of mutation in maintaining quantitative genetic variation. Genet Res 80:31-46

Chapuisat M (1996) Characterization of microsatellite loci in Formica lugubris $B$ and their variability in other ant species. Mol Ecol 5:599-601

Chapuisat M, Bocherens S, Rosset H (2004) Variable queen number in ant colonies: no impact on queen turnover, inbreeding, and population genetic differentiation in the ant Formica selysi. Evolution 58:1064-1072

Costa JT, Ross KG (2003) Fitness effects of group merging in a social insect. Proc R Soc Lond B 270:1697-1702

Crozier RH, Page RE (1985) On being the right size: male contributions and multiple mating in social Hymenoptera. Behav Ecol Sociobiol 18:105-115

Franks NR (1985) Reproduction, foraging efficiency and worker polymorphism in army ants. In: Lindauer M, Hölldobler B (eds) Experimental behavioral ecology and sociobiology. Gustav Fischer Verlag, Stuttgart, pp 91-107

Fraser VS, Kaufmann B, Oldroyd BP, Crozier RH (2000) Genetic influence on caste in the ant Camponotus consobrinus. Behav Ecol Sociobiol 47:188-194

Goodisman M, Ross KG (1996) Relationship of queen number and worker size in polygyne colonies of the fire ant Solenopsis invicta. Ins Soc 43:303-307

Goodisman MAD, Crozier RH (2003) Association between caste and genotype in the termite Mastotermes darwiniensis Froggatt (Isoptera : Mastotermitidae). Aust J Entomol 42:1-5

Greenberg L, Fletcher DJC, Vinson SB (1985) Differences in worker size and mound distribution in monogynous and polygynous colonies of the fire ant Solenopsis invicta Buren. J Kans Entomol Soc 58:9-18

Gyllenstrand N, Gertsch PJ, Pamilo P (2002) Polymorphic microsatellite DNA markers in the ant Formica exsecta. Mol Ecol Notes 2:67-69

Hölldobler B, Wilson EO (1990) The ants. Springer-Verlag, Berlin

Hughes WOH, Sumner S, van Borm S, Boomsma JJ (2003) Worker caste polymorphism has a genetic basis in Acromyrmex leaf-cutting ants. Proc Natl Acad Sci USA 100:9394-9397

Jones JC, Myerscough MR, Graham S, Oldroyd BP (2004) Honey bee nest thermoregulation: diversity promotes stability. Science 305:402-404

Julian GE, Fewell JH (2004) Genetic variation and task specialisation in the desert leaf-cutter ant, Acromyrmex versicolor. Anim Behav 68:1-8

Liu FH, Smith SM (2000) Estimating quantitative genetic parameters in haplodiploid organisms. Heredity 85:373-382

Manly BFJ (1997) Randomization, bootstrap and Monte Carlo methods in biology, 2nd edn. Chapman and Hall, London

Oster GF, Wilson EO (1978) Caste and ecology in the social insects. Princeton University Press, Princeton, NJ

Page RE, Erber J (2002) Levels of behavioral organization and the evolution of division of labor. Naturwissenschaften 89:91-106 
Page RE, Robinson GE, Fondrk MK, Nasr ME (1995) Effects of worker genotypic diversity on honey bee colony development and behavior (Apis mellifera L.). Behav Ecol Sociobiol 36:387-396

Pisarski B (1981) Intraspecific variations in ants of the genus Formica. In: Howse PE, Clement J-L (eds) Biosystematics of social insects. Academic Press, London and New York, pp 17-25

Porter SD, Tschinkel WR (1985a) Fire ant polymorphism (Hymenoptera: Formicidae): factors affecting worker size. Ann Entomol Soc Am 78:381-386

Porter SD, Tschinkel WR (1985b) Fire ant polymorphism: the ergonomics of brood production. Behav Ecol Sociobiol 16:323-336

Robinson GE (1992) Regulation of division of labor in insect societies. Annu Rev Entomol 37:637-665

Rüppell O, Heinze J, Holldobler B (2001) Complex determination of queen body size in the queen size dimorphic ant Leptothorax rugatulus (Formicidae : Hymenoptera). Heredity 87:33-40

Snyder LE (1992) The genetics of social behavior in a polygynous ant. Naturwissenschaften 79:525-527

Sokal RR, Braumann CA (1980) Significance tests for coefficients of variation and variability profiles. Syst Zool 29:50-66

Sokal RR, Rohlf FJ (1995) Biometry. The principles and practice of statistics in biological research, 3rd edn. Freeman, New York

Stuart RJ, Page RE (1991) Genetic component to division of labor among workers of a leptothoracine ant. Naturwissenschaften $78: 375-377$
Sundström L (1995) Sex allocation and colony maintenance in monogyne and polygyne colonies of Formica truncorum (Hymenoptera, Formicidae): the impact of kinship and mating structure. Am Nat 146:182-201

Turelli M, Barton NH (2004) Polygenic variation maintained by balancing selection: pleiotropy, sex-dependent allelic effects and $\mathrm{GxE}$ interactions. Genetics 166:1053-1079

Wang JL (2004) Sibship reconstruction from genetic data with typing errors. Genetics 166:1963-1979

Waser NM (1998) Task-matching and short-term size shifts in foragers of the harvester ant, Messor pergandei (Hymenoptera: Formicidae). J Insect Behav 11:451-462

Wetterer JK (1999) The ecology and evolution of worker sizedistribution in leaf-cutting ants (Hymenoptera: Formicidae). Sociobiology 34:119-144

Wheeler DE (1991) The developmental basis of worker caste polymorphism in ants. Am Nat 138:1218-1238

Wilson EO (1968) The ergonomics of caste in the social insects. Am Nat 102:41-66

Wilson EO (1971) The insect societies. Harvard University Press, Cambridge, MA

Wilson EO (1976) Behavioral discretization and the number of castes in an ant species. Behav Ecol Sociobiol 1:141154

Wilson EO (1980) Caste and division of labor in leaf-cutter ants (Hymenoptera: Formicidae: Atta). II. The ergonomic optimization of leaf cutting Atta sexdens. Behav Ecol Sociobiol 7:157-165 